\title{
Surgical management of spinal metastases involving the cervicothoracic junction: results of a multicenter, European observational study
}

\author{
*Vanessa Hubertus, MD, ${ }^{1}$ Jens Gempt, MD, ${ }^{2}$ Michelle Mariño, MD, ${ }^{1}$ Björn Sommer, MD, ${ }^{3}$ \\ Sven O. Eicker, MD, ${ }^{4}$ Martin Stangenberg, MD, ${ }^{5}$ Marc Dreimann, MD, ${ }^{5}$ Insa Janssen, MD, ${ }^{6}$ \\ Christoph Wipplinger, MD, ${ }^{7}$ Arthur Wagner, MD, ${ }^{2}$ Nicole Lange, MD, ${ }^{2}$ Ann-Kathrin Jörger, MD, ${ }^{2}$ \\ Marcus Czabanka, MD, ${ }^{1}$ Veit Rohde, MD, ${ }^{3}$ Karl Schaller, MD, ${ }^{6}$ Claudius Thomé, MD, ${ }^{7}$ \\ Peter Vajkoczy, MD, ${ }^{1}$ Julia S. Onken, MD, ${ }^{1}$ and Bernhard Meyer, MD²
}

\begin{abstract}
'Department of Neurosurgery, Charité-Universitätsmedizin Berlin, Freie Universität Berlin, Humboldt-Universität zu Berlin and Berlin Institute of Health, Berlin; ${ }^{2}$ Department of Neurosurgery, School of Medicine, Klinikum rechts der Isar, Technical University of Munich; ${ }^{3}$ Department of Neurosurgery, Universitätsmedizin Göttingen; ${ }^{4}$ Department of Neurosurgery and Interdisciplinary University Spine Center, Universitätsklinikum Hamburg-Eppendorf, Hamburg; ${ }^{5}$ Department of Trauma and Orthopedic Surgery and Interdisciplinary University Spine Center, Universitätsklinikum Hamburg-Eppendorf, Hamburg, Germany; ${ }^{6}$ Department of Neurosurgery, Hôpitaux Universitaires de Genève, Switzerland; and 'Department of Neurosurgery, Medizinische Universität Innsbruck, Innsbruck, Austria
\end{abstract}

OBJECTIVE Surgical management of spinal metastases at the cervicothoracic junction (CTJ) is highly complex and relies on case-based decision-making. The aim of this multicentric study was to describe surgical procedures for metastases at the CTJ and provide guidance for clinical and surgical management.

METHODS Patients eligible for this study were those with metastases at the CTJ (C7-T2) who had been consecutively treated in 2005-2019 at 7 academic institutions across Europe. The Spine Instability Neoplastic Score, neurological function, clinical status, medical history, and surgical data for each patient were retrospectively assessed. Patients were divided into four surgical groups: 1) posterior decompression only, 2) posterior decompression and fusion, 3) anterior corpectomy and fusion, and 4) anterior corpectomy and $360^{\circ}$ fusion. Endpoints were complications, surgical revision rate, and survival.

RESULTS Among the 238 patients eligible for inclusion this study, 37 were included in group 1 (15\%), 127 in group 2 $(53 \%), 18$ in group $3(8 \%)$, and 56 in group $4(24 \%)$. Mechanical pain was the predominant symptom (79\%, 189 patients). Surgical complications occurred in $16 \%$ (group 1), $20 \%$ (group 2), $11 \%$ (group 3), and 18\% (group 4). Of these, hardware failure (HwF) occurred in $18 \%$ and led to surgical revision in 7 of 8 cases. The overall complication rate was $34 \%$. Inhospital mortality was $5 \%$.

CONCLUSIONS Posterior fusion and decompression was the most frequently used technique. Care should be taken to choose instrumentation techniques that offer the highest possible biomechanical load-bearing capacity to avoid HwF. Since the overall complication rate is high, the prevention of in-hospital complications seems crucial to reduce in-hospital mortality. https://thejns.org/doi/abs/10.3171/2021.2.FOCUS201067

KEYWORDS cervicothoracic junction; NOMS criteria; SINS; spinal metastases; spinal stabilization; surgical management

$\mathrm{F}$ OR the treatment of spinal metastases, multimodal concepts consisting of surgical therapy with or without radio-/chemo-/immunotherapy have proven successful. However, surgical management of spinal metastases is often complex and relies on case-based decision- making. The choice of surgical procedure depends on patient clinical status and neurological symptoms as well as on biomechanical considerations and spinal stability. Decisive factors that also influence the extent of surgical intervention include tumor sensitivity to radiation and chemo-

ABBREVIATIONS ASA = American Society of Anesthesiologists; $C T J=$ cervicothoracic junction; $\mathrm{HwF}=$ hardware failure; $\mathrm{ICU}=$ intensive care unit; $\mathrm{KPS}=\mathrm{Karnofsky}$ Performance Status; NOMS = neurologic, oncologic, mechanical, and systemic; SCC = spinal cord compression; SINS = Spine Instability Neoplastic Score; SSI = surgical site infection.

SUBMITTED December 15, 2020. ACCEPTED February 24, 2021.

INCLUDE WHEN CITING DOI: 10.3171/2021.2.FOCUS201067.

*J.S.O. and B.M. contributed equally and share senior authorship of this work. 
therapy, systemic tumor burden, and the patient's clinical performance status and proposed life expectancy. ${ }^{1,2}$ Clinical scores are intended to simplify therapeutic decisionmaking. The Spine Instability Neoplastic Score (SINS) attempts to address biomechanical considerations; ${ }^{3}$ however, the SINS does not translate into concrete treatment recommendations..$^{4-7}$ The applicability of prognostic scores such as the Tomita or Tokuhashi scores is limited, as the scores were validated before the era of new, life-prolonging therapies such as immunotherapy. ${ }^{8-13}$ The development of evidence-based treatment guidelines in metastatic spine disease is also hampered by the diversity of clinical and imaging findings, which makes the design of prospective randomized clinical trials difficult. Therefore, larger case series are of great scientific interest. ${ }^{13}$ Very little is published about the management of spinal metastases at the cervicothoracic junction (CTJ). As part of the junctional spine, the CTJ is prone to spinal instability and neurological deficits. Case series have reported the occurrence of neurological deficits, with metastases in this region in up to $100 \%$ of cases. ${ }^{7,14-18}$ On the basis of the current literature, spinal fusion via posterior stabilization in addition to posterior or anterior decompression at the CTJ is strongly recommended. ${ }^{7}$ However, these recommendations rely on expert opinions. Level I recommendations regarding the choice of surgical strategies at the CTJ are lacking.

With the present study, we aimed to identify current treatment strategies and characterize decision-making in this field. We analyzed the outcomes of different surgical strategies by comparing those undertaken in 238 patients with spinal metastases at the CTJ in a multicenter, international, European observational trial. Patients underwent posterior decompression only (group 1), posterior decompression and fusion (group 2), anterior corpectomy and fusion (group 3), or anterior corpectomy and $360^{\circ}$ fusion (group 4). Primary endpoints were surgical and medical complications and surgical revision rate, and the secondary endpoint was postoperative survival.

\section{Methods \\ Clinical Data}

Consecutive data sets of patients who, between 2005 and 2019, had undergone surgical treatment for spinal metastases at the CTJ, defined as C7-T2, were retrospectively evaluated at 7 academic institutions across Europe. Besides collecting demographic data, we assessed neurological function using the modified Japanese Orthopaedic Association scale and the American Spinal Injury Association Impairment Scale and recorded clinical status according to the Karnofsky Performance Status (KPS) and American Society of Anesthesiologists (ASA) classification. Mechanical pain intensity was assessed using the visual analog scale. Systemic tumor burden and comorbidities were assessed by applying the neurologic, oncologic, mechanical, and systemic (NOMS) decision framework. ${ }^{2}$ Furthermore, the site of epidural metastasis and the SINS were assessed. Surgical information included duration of surgery, surgical approach, screw fixation techniques, and use of spinal navigation techniques. Outcome data included length of hospital stay, length of stay on an intensive care unit (ICU), discharge from the regular ward, postoperative complications, surgical revision rate, hardware failure $(\mathrm{HwF})$, secondary deformity, local tumor recurrence, and postoperative survival.

\section{Ethics Statement}

This study was conducted according to the ethical principles of medical research involving human subjects according to the Declaration of Helsinki. The clinical data were assessed retrospectively and anonymized for patient confidentiality. Written patient consent was not required. Ethics approval was granted by the institutional ethics boards of the institutions participating in the study.

\section{Statistical Analysis}

Statistical analysis was performed using GraphPad Prism 8 (GraphPad Software Inc.). For statistical analysis, one-way ANOVA combined with Bonferroni's multiple comparison test was used. Kaplan-Meier survival curves were obtained, and differences in postoperative survival were tested for statistical significance using the log-rank test. Significance level was set at $\mathrm{p}<0.05$.

\section{Results}

\section{Study Cohort and Clinical Presentation}

Two hundred thirty-eight patients were included in the study. Mean age at diagnosis of spinal metastasis was 63 (14-92) years. The most common underlying malignancies were lung, breast, and prostate cancer. Most cases were treated with posterior decompression and fusion (group 2, $53 \%$ ), and $24 \%$ of patients underwent anterior corpectomy plus $360^{\circ}$ fusion (group 4). Posterior decompression alone (group 1) was performed in 15\%, and anterior corpectomy plus fusion (group 3) was performed in $8 \%$. Overall, $80 \%$ of patients presented with axial pain. Acute neurological deficits prompted surgery in $18 \%$ of patients. Acute or chronic neurological deficits were observed in $50 \%$ of patients. At initial presentation, axial pain was most commonly found in group 4 at $89 \%$ of patients (Table 1). In patients treated with decompression only (group 1), the SINS was the lowest at 7 points (Table 2). In the groups treated with spinal stabilization (groups 2-4), the SINSs were similar at 11 points (groups 2 and 3 ) and 12 points (group 4). The groups differed in tumor localization: in metastases treated with decompression only (group 1), the SINS was stable and tumor localization was mostly epidural. In groups $2-4$, bone infiltration was common. Thus, the SINS was potentially unstable and epidural tumor growth occurred from anteriorly (group 3 ) or posteriorly (group 2 ) and often circumferentially in group 4 . In group 2 , a mean of two segments were affected by metastasis, and in groups 1,3 , and 4, a mean of one segment was affected. Groups did not differ significantly in their physical status as reflected by their ASA class (mean of 3). Tumor burden with additional metastases was the highest in group $1(95 \%)$ and lowest in group 4 (77\%). The NOMS criteria are displayed in Table 2.

\section{Surgical Management}

Clinical and imaging characteristics for each surgical 
TABLE 1. Clinical presentation of patients who underwent surgical treatment for spinal metastases at the CTJ

\begin{tabular}{|c|c|c|c|c|c|c|}
\hline Variable & All & Group 1 & Group 2 & Group 3 & Group 4 & p Value \\
\hline Axial pain, no. (\%) & $189(79 \%)$ & $18(49 \%)$ & $106(83 \%)$ & $15(83 \%)$ & $50(89 \%)$ & $<0.0001^{*}$ \\
\hline Acute deficits in last $24 \mathrm{hrs}$, no. (\%) & $42(18 \%)$ & $10(27 \%)$ & $24(19 \%)$ & $2(11 \%)$ & $6(11 \%)$ & 0.1922 \\
\hline \multicolumn{7}{|l|}{ AIS grade, no. $(\%)$} \\
\hline A & $11(5 \%)$ & $5(14 \%)$ & $6(5 \%)$ & $0(0 \%)$ & $0(0 \%)$ & \\
\hline $\mathrm{C}$ & $35(15 \%)$ & $8(22 \%)$ & $20(16 \%)$ & $1(6 \%)$ & $6(11 \%)$ & \\
\hline$D$ & $65(27 \%)$ & $6(16 \%)$ & $33(26 \%)$ & $9(50 \%)$ & $17(30 \%)$ & \\
\hline $\mathrm{E}$ & $120(50 \%)$ & $18(49 \%)$ & $63(50 \%)$ & $8(44 \%)$ & $31(55 \%)$ & \\
\hline Any neurological deficit, no. (\%) & $120(50 \%)$ & $18(49 \%)$ & $63(50 \%)$ & $8(44 \%)$ & $31(55 \%)$ & $0.0182 \dagger$ \\
\hline Motor deficits, no. (\%) & $91(38 \%)$ & $17(46 \%)$ & $48(38 \%)$ & $6(33 \%)$ & $20(36 \%)$ & 0.9795 \\
\hline Sensory deficits, no. (\%) & $94(39 \%)$ & $15(41 \%)$ & $53(42 \%)$ & $7(39 \%)$ & $19(34 \%)$ & 0.9434 \\
\hline Mean KPS $( \pm$ SD) & $72( \pm 17)$ & $70( \pm 17)$ & $71( \pm 18)$ & $82( \pm 12)$ & $75( \pm 15)$ & $0.0220 \dagger$ \\
\hline Mean ASA class ( \pm SD) & $3( \pm 1)$ & $3( \pm 1)$ & $3( \pm 1)$ & $3( \pm 1)$ & $3( \pm 1)$ & 0.5425 \\
\hline
\end{tabular}

group were evaluated to comprehensively illustrate the therapy decisions. Fig. 1 illustrates representative cases assigned to the four surgical groups. Data on duration of surgery, SINS, duration of hospitalization, and discharge are displayed in Fig. 2.

\section{Posterior Decompression Only: Group 1}

Patients treated with posterior decompression without instrumentation had no unstable bone lesions, which is reflected by the low incidence of axial pain $(50 \%)$ and stable SINS (mean \pm SD, $7 \pm 3$ points). The indication for surgery was neurological deficits due to spinal cord compression (SCC). Decompression was performed via laminectomy (mean $1 \pm 0.6$ segments). Mean duration of surgery was 129 (60-357) minutes. In these patients, the systemic tumor burden was highest $(95 \%$ with metastases other than spine) and the worst KPS (70 \pm 17$)$. The surgical complication rate was lowest in this group at $16 \%$ (6 patients). Of these, 3 patients developed surgical site infections (SSIs) and 3 patients experienced secondary deformity during follow-up. No surgical revision was required in these cases, as the patients either had no symptoms or were at an advanced tumor stage.

\section{Posterior Decompression and Fusion: Group 2}

Most cases $(127,53 \%)$ included in the study were treated with posterior decompression and fusion. Mean SINS was $11( \pm 3)$ points. In these patients, metastatic epidural SCC mostly occurred from posteriorly with the anterior column remaining aligned. Mean duration of surgery was 239 (68-534) minutes. Mean number of surgically fused segments was $5( \pm 2)$. Surgical fusion was performed via posterolateral instrumentation. In $40 \%$ of cases, lateral mass screws were placed in the cervical segment and pedicle screws were placed in the thoracic segment. In $56 \%$ of cases, pedicle screws were placed in the cervical and thoracic segments. Spinal navigation was utilized in 71 cases $(56 \%)$. When pedicle screws were placed, spinal navigation was utilized in 45 cases (63\%). Surgery was performed using fluoroscopic control when spinal

TABLE 2. The NOMS criteria of the surgical groups

\begin{tabular}{lcccccc}
\hline \multicolumn{1}{c}{ Variable } & All & Group 1 & Group 2 & Group 3 & Group 4 & $p$ Value \\
\hline Mean no. of vertebrae involved in metastasis $( \pm$ SD) & $1( \pm 1)$ & $1( \pm 1)$ & $2( \pm 1)$ & $1( \pm 1)$ & $1( \pm 1)$ & $0.0102^{*}$ \\
\hline Mean SINS $( \pm$ SD) & $10( \pm 4)$ & $7( \pm 3)$ & $11( \pm 3)$ & $11( \pm 2)$ & $12( \pm 3)$ & $<0.0001 \dagger$ \\
\hline Metastases other than spine, no. $(\%)$ & $204(86 \%)$ & $35(95 \%)$ & $111(87 \%)$ & $15(83 \%)$ & $43(77 \%)$ & $0.0407^{*}$ \\
\hline The NOMS criteria based on the work of Laufer et al., 2013. ${ }^{2}$ & & & & & \\
\\
${ }^{*} p<0.05$. \\
$+p<0.0001$.
\end{tabular}




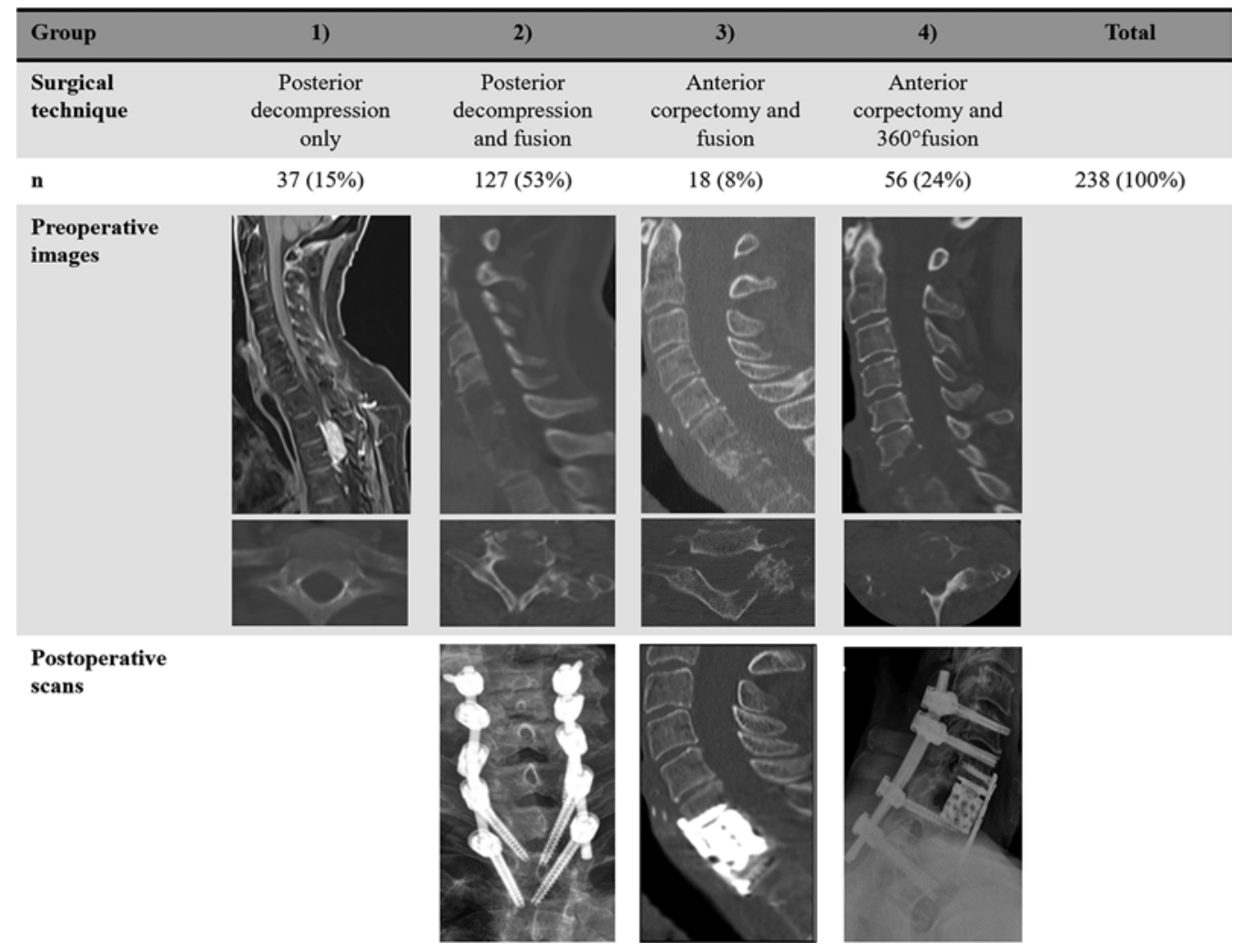

FIG. 1. Distribution of four surgical groups and corresponding case illustration with pre- and postoperative images.

navigation was not utilized. Postoperative complications in general were highest in this group (43\%), and surgical complications occurred in $20 \%$ of patients. SSI and $\mathrm{HwF}$ were the leading causes of revision surgery. All 7 cases with HwF during follow-up in this group were related to loosening and/or secondary dislocation of monocortical lateral mass screws in the cervical spine (Fig. 3). No HwF was reported in patients with pedicle screws or bicortically placed lateral mass screws in the cervical spine at the 10-month follow-up. Among the cases of HwF, 6 of 7 patients underwent revision surgery. The 7 th patient did not undergo surgical revision because of their highly palliative condition.

\section{Anterior Corpectomy and Fusion: Group 3}

Only $8 \%$ of the study cohort was treated with anterior corpectomy and fusion. In these cases, metastatic epidural SCC usually occurred from anteriorly in patients with a mean SINS of $11( \pm 2)$ points. An isolated anterior approach was chosen in mono- or bisegmental diseases with unilateral or no involvement of the posterior column. All patients in this group underwent a one-level corpectomy with implantation of an anterior distraction device via an anterior approach. Additionally, all patients underwent three-level stabilization via anterior plate fixation (one level above and below the corpectomy). The mean duration of surgery was 166 (66-280) minutes. All surgeries in this group were performed using fluoroscopic control. Surgical complications occurred in 2 patients (11\%). One of them developed a paravertebral hematoma, while the other showed dislocation of the vertebral body replacement during follow-up (monosegmental disease). Both patients underwent revision surgery. Injury to the recurrent laryngeal nerve with symptoms like severe hoarseness or permanent dysphagia was not reported.

\section{Anterior Corpectomy and $360^{\circ}$ Fusion: Group 4}

Twenty-four percent of patients were treated with anterior corpectomy and $360^{\circ}$ fusion. Patients typically suffered from a potentially unstable metastatic lesion (mean SINS $12 \pm 3$ points) affecting both the anterior and posterior column. Anterior corpectomy and plate fixation were performed in the same fashion as in group 3. Mean duration of surgery was 305 (119-752) minutes. A twostaged surgical approach was chosen in 58\% of the cases, either with anterior corpectomy and plate fixation or with posterolateral instrumentation first. The mean number of surgically fused segments was $4(\mathrm{SD} \pm 2)$. The surgical complication rate was $18 \%$. SSI was the leading cause for revision surgery in this group (9 cases). One case required revision surgery because of primary pedicle screw malpositioning in the cervical segment. No $\mathrm{HwF}$ was seen during follow-up (mean 13 months). Spinal navigation was utilized in 39 cases $(70 \%)$. All other cases were performed with fluoroscopic control.

\section{Advances in the Surgical Management of Metastatic Spine Diseases Over Time}

In a further step, we analyzed whether the surgical 
A

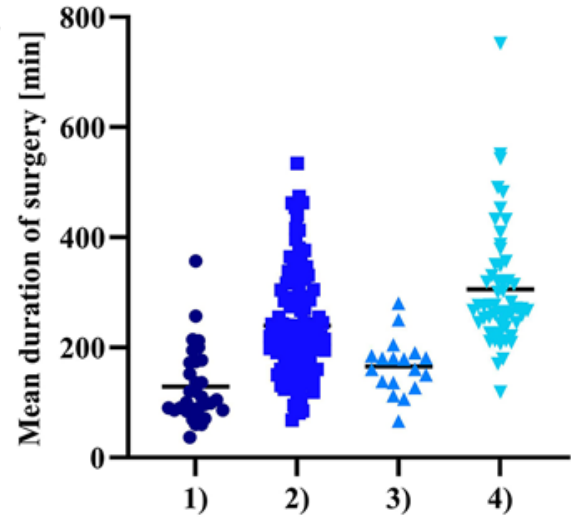

C

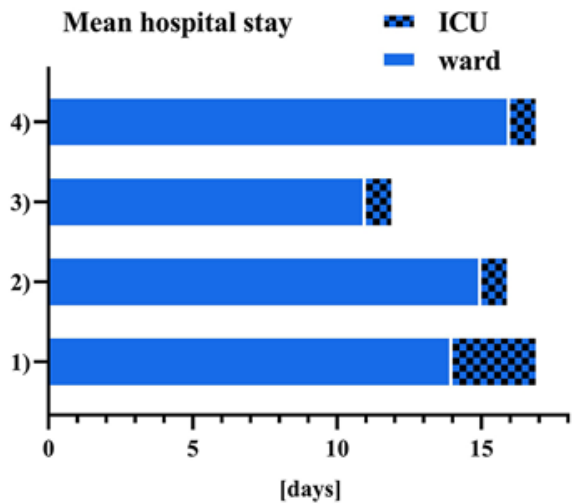

B
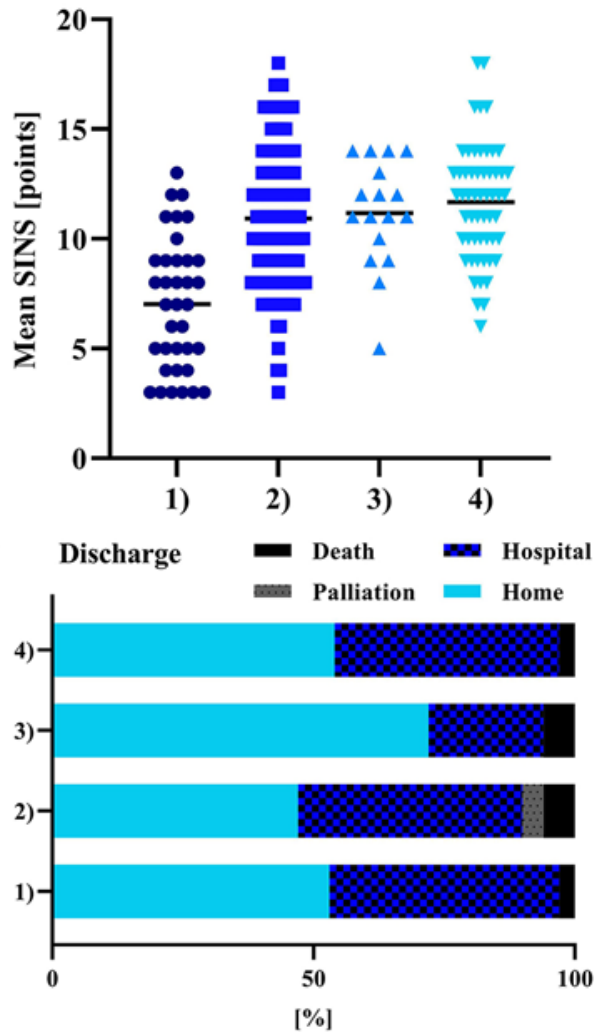

FIG. 2. A: Mean duration of surgery in minutes. Bonferroni's multiple comparison test showed significant differences between groups ( $<$ 0.0001) except group 1 versus 3 and group 2 versus 3 . B: With regard to the mean SINS, Bonferroni's multiple comparison test showed significant differences between groups 1 and $2(p<0.0001)$ and between groups 3 and $4(p<0.0001)$. C: Mean hospital stay with proportions of stay on the neurosurgical ward and the ICU in days. D: Percentage discharged from the neurosurgical ward.

strategies used to treat CTJ metastases have changed over time. Here, we found a significant increase in the use of $360^{\circ}$ instrumentation techniques (group 4) and posterior fusion alone (group 2). Starting in 2012, a trend toward the more frequent use of spinal navigation techniques became apparent. A corresponding increase of pedicle screw placement in the cervical spine was also observed. Remarkably, the likelihood of surgical complications such as screw malpositioning and $\mathrm{HwF}$ in groups 2 and 4 did not increase over the years (Fig. 4).

\section{Complication Rate and Outcome Data}

The overall complication rate during the hospital stay was high at $34 \%, 18 \%$ of which was surgical complications and $17 \%$ medical complications. Revision surgery was required in $86 \%$ of the surgical complication cases. The main reasons for revision surgery were SSI (69\%) and postoperative hematoma (10\%). The most common medical complications included pneumonia, sepsis, cardiac arrhythmia, thrombosis, and pulmonary artery embolisms. The highest rate of surgical complications was found in group 2 (20\%), followed by group 4 (18\%). In-hospital mortality was $5 \%$ and was related to postoperative medical complications such as pulmonary artery embolism, multiple organ failure, and myocardial infarction (Table 3 ). No deaths were reported to be causally related to any surgical intervention. Postoperative in-hospital medical complications had a significant impact on in-hospital mortality whereas surgical complications did not (Fig. 5A).

The mean hospital stay was 15 days ( \pm 9 days) and did not vary significantly between surgical groups. The mean stay on an ICU was 1 day ( \pm 4 days) and did not differ significantly between surgical groups. Among all groups, $51 \%$ of patients were discharged home, $41 \%$ were directly transferred to further medical or (radio-)oncological treatment, and $7 \%$ were transferred to palliative treatment or died. The occurrence of surgical complications prompting surgical revision did prolong hospitalization time significantly in group 1 ( $40 \pm 17$ days instead of $10 \pm 6$ days, $\mathrm{p}<0.0001$ ). Hospitalization time was not significantly prolonged by the occurrence of surgical complications in groups $2-4$.

Following surgical treatment of spinal metastasis, $87 \%$ of patients received adjuvant treatment with radiation $(78 \%)$ or systemic antitumor treatment (chemotherapy $58 \%$, immunotherapy 12\%). The mean follow-up was 13 months in group 1, 6 months in group 2, 14 months in group 3 , and 13 months in group 4 . The mean overall survival was highest in group 4 at 47 months, followed by group 1 at 36 months (1 patient with metastatic breast cancer survived 104 months), followed by group 2 at a mean survival of 9 months and group 3 at 7 months. In terms of 

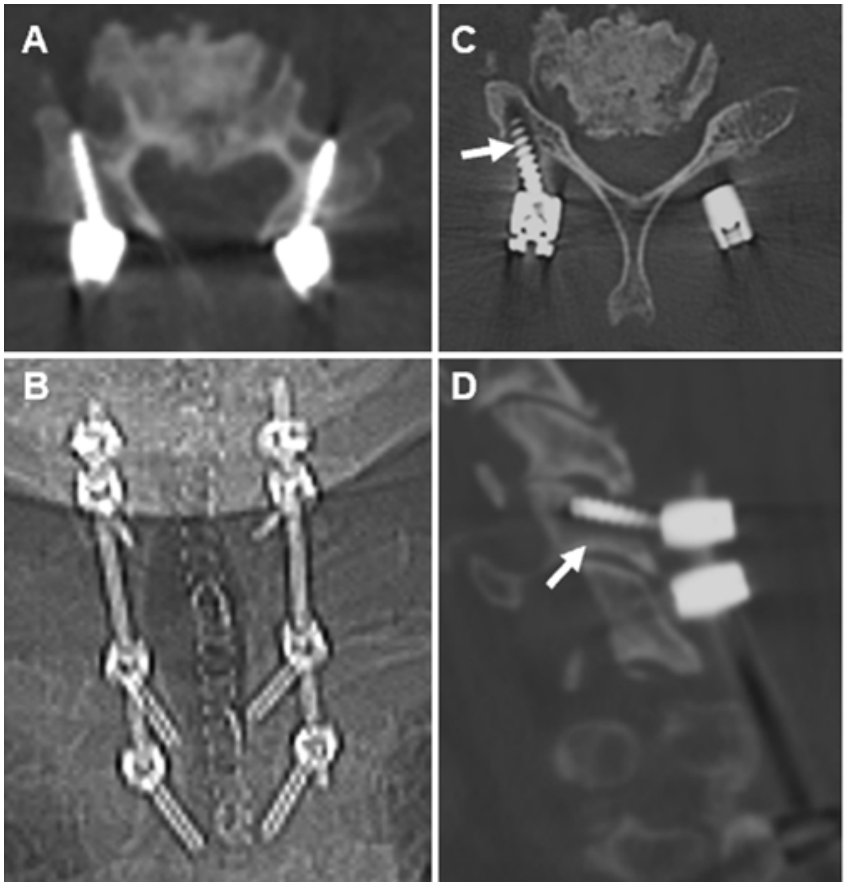
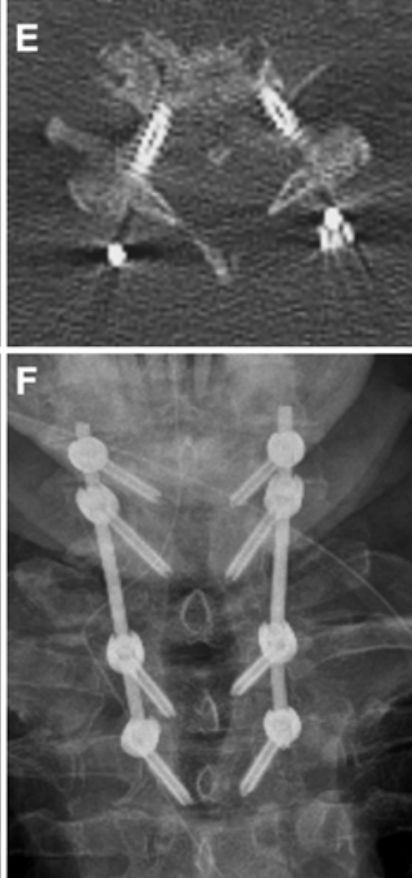

FIG. 3. Case illustration of a 75-year-old man suffering from metastatic non-small cell lung carcinoma with metastatic infiltration of T1 (SINS 10 points) without neurological deficits. A and B: Surgical management analog group 2, with dorsal decompression and stabilization at C6-T3 (lateral mass screws at C6, bicortically placed on the left, monocortically placed on the right; pedicle screws at C7 and T2-3). C and D: At the 10-month follow-up after the index surgery, it was noted that loosening and partial pullout of the right lateral mass screw at $\mathrm{C} 6$ had occurred (white arrows), and the patient presented with mechanical pain. $\mathrm{E}$ and F: During surgical revision, pedicle screws were placed in $\mathrm{C} 6$ and $\mathrm{C} 7$ bilaterally using spinal navigation. The overall postoperative follow-up in this patient after the index surgery was 32 months, and no neurological deficit or mechanical pain has occurred since revision surgery.

A
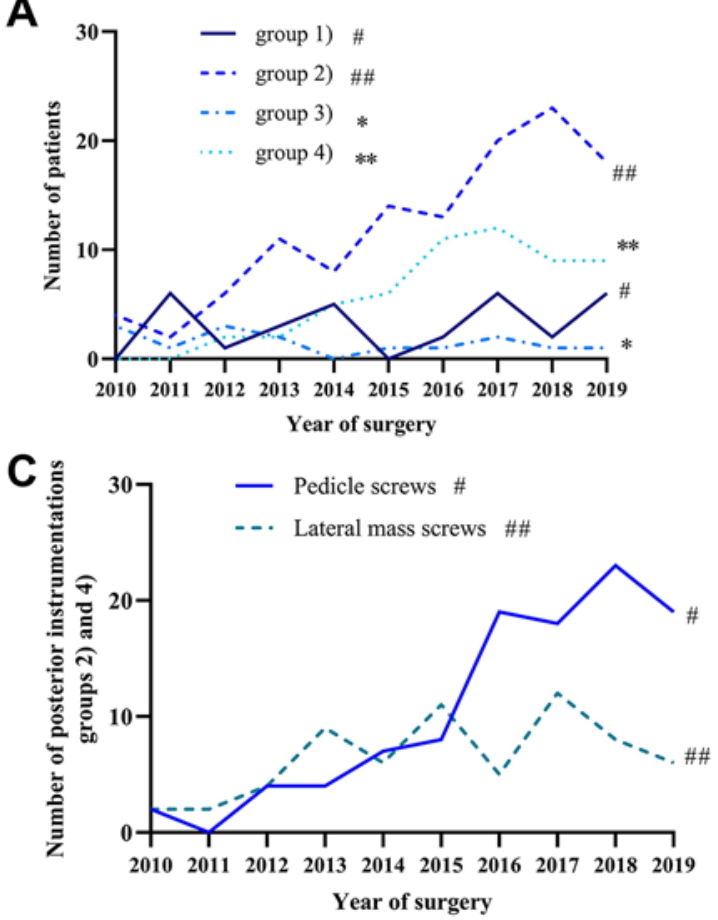

B

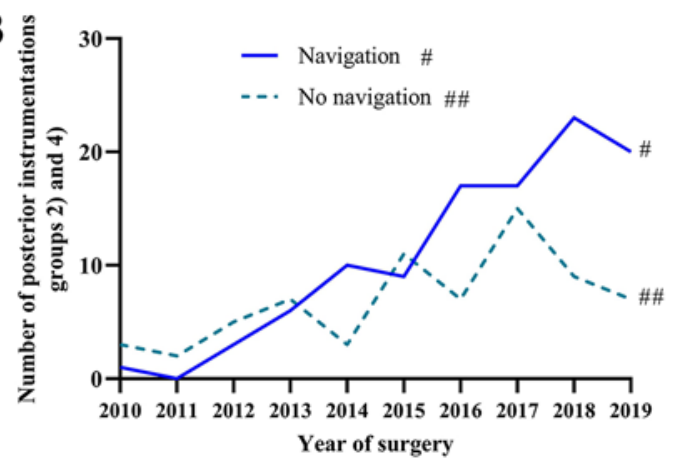

D

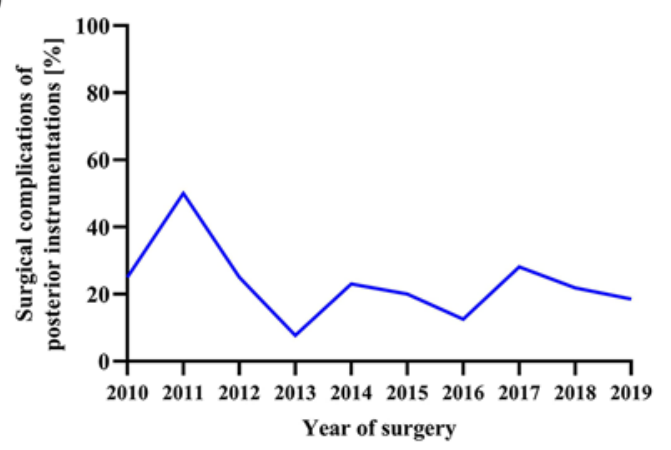

FIG. 4. Advances in the surgical management of metastatic spine diseases over time. Increasing numbers of patients treated according to groups 2 and 4 (A). Increasing use of spinal navigation over the last 10 years (B), which correlates with the increasing use of pedicle screws in the cervical segment in group 2 (C). Surgical complication rate remained constant over the years (D). 
TABLE 3. Data on postoperative complications

\begin{tabular}{|c|c|c|c|c|c|c|}
\hline Variable & All & Group 1 & Group 2 & Group 3 & Group 4 & p Value \\
\hline Postop complication & $82(34 \%)$ & $6(16 \%)$ & $54(43 \%)$ & $4(22 \%)$ & $18(32 \%)$ & $0.0260^{*}$ \\
\hline Surgical complication (\% of all complications) & $44(18 \%)$ & $6(16 \%)$ & $26(20 \%)$ & $2(11 \%)$ & $10(18 \%)$ & 0.1104 \\
\hline \multicolumn{7}{|l|}{ During hospital stay } \\
\hline SSI & & $3(50 \%)$ & $18(69 \%)$ & 0 & $9(90 \%)$ & \\
\hline Malpositioning of hardware & & 0 & 0 & 0 & $1(10 \%)$ & \\
\hline Hematoma & & 0 & $1(4 \%)$ & $1(50 \%)$ & 0 & \\
\hline \multicolumn{7}{|l|}{ During follow-up } \\
\hline Kyphosis & & $3(50 \%)$ & 0 & 0 & 0 & \\
\hline $\mathrm{HwF}$ & & 0 & $7(27 \%)$ & $1(50 \%)$ & 0 & \\
\hline In-hospital mortality & $12(5 \%)$ & $1(3 \%)$ & $8(6 \%)$ & $1(6 \%)$ & $2(4 \%)$ & 0.7792 \\
\hline
\end{tabular}

survival rate related to the underlying malignancy, mean overall survival was significantly influenced by the type of primary tumor, with the lowest survival rate found in metastatic lung cancer (Fig. 5B).

\section{Discussion}

With this study, we provide data on the surgical management of metastasis at the CTJ by comparing different surgical strategies in an international, European multicenter cohort. In line with previous studies, the overall complication rate in our cohort is high at $34 \% .{ }^{15-19} \mathrm{We}$ observed that medical complications in particular have a significant impact on in-hospital mortality. These data emphasize the importance of the prevention of postoperative complications.

By analyzing patient characteristics, risks, and advantages of different surgical procedures, we drew the following conclusions. Posterior decompression alone seems feasible when spinal stability is preserved. A certain risk for secondary deformity needs to be considered. In cases with advanced metastatic disease, this approach may be advantageous because of its short duration and comparably low surgical complication rate. Close radiological follow-up is indicated for these patients to detect secondary deformity at an early stage.

Little data exist for an isolated anterior approach to the CTJ in metastatic spine disease. ${ }^{7}$ We showed that anterior corpectomy and fusion presents a robust construct with low rates of $\mathrm{HwF}$ and almost half as many surgical complications than other instrumentation techniques. Previously published data on the long-term follow-up of mono- or bisegmental anterior corpectomy in degenerative spinal disease suggest that a $360^{\circ}$ construct is preferred to avoid $\mathrm{HwF}^{20}$ The reduced life expectancy of patients with metastatic spine disease may be a reason that HwF was not seen in our cohort. On the basis of these data, we recommend that in cases with anterior corpectomy and fusion only, radiological follow-up should be closely maintained.

In line with the literature, most of our cases were treated with complex procedures like multilevel posterior decompression and fusion (with or without $360^{\circ}$ reconstruction). These procedures were associated with considerably
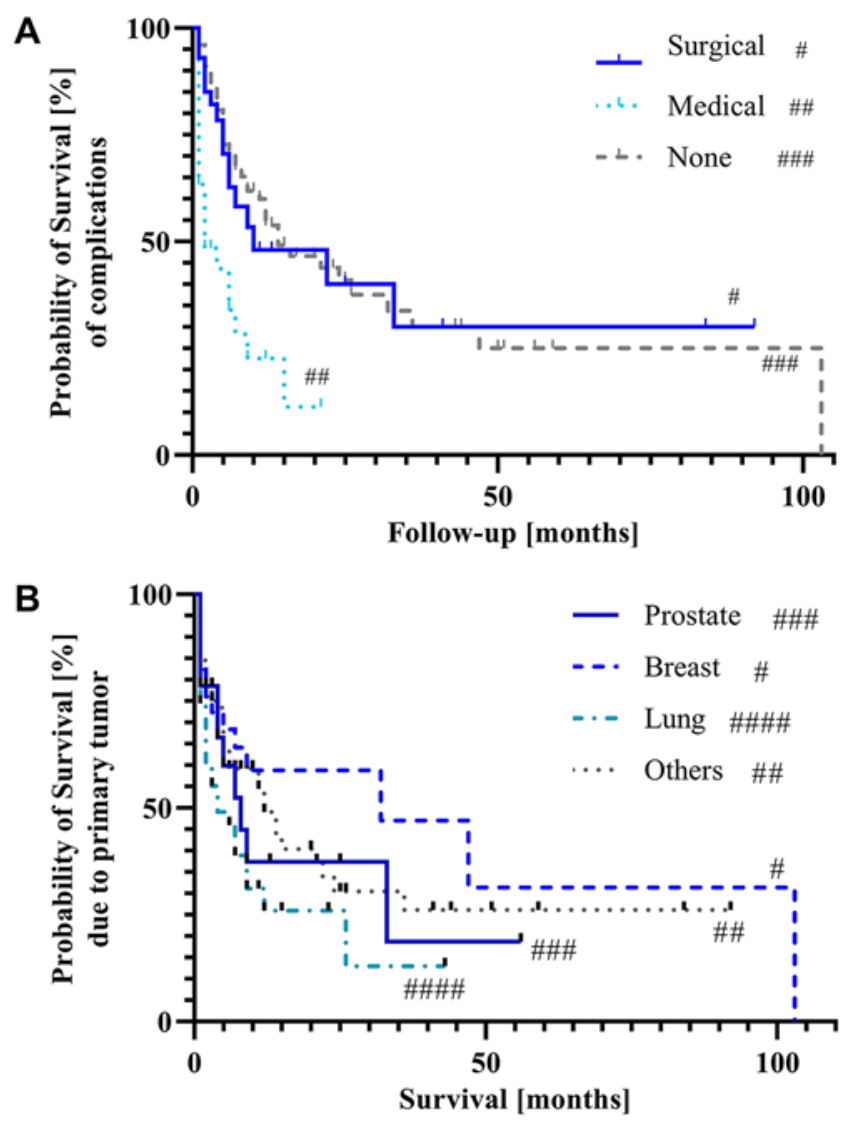

FIG. 5. A: Probability of postoperative survival with and without the occurrence of postoperative surgical or medical complications. Bonferroni's multiple comparison test showed significant differences in survival comparing no complications versus medical complications (\#\#\# vs \#\#, $p=0.0008$ ) and surgical versus medical complications (\# vs \#\#, $p<$ 0.0001). B: Probability of survival due to primary tumor. Bonferroni's multiple comparison test showed significant differences in survival comparing breast versus lung cancer (\# vs \#\#\#, $p=0.0183$ ) and lung cancer versus others (\#\#\# vs \#\#, $p=0.0253$ ). All other comparisons between groups were not significant. 
high complication rates in terms of SSI, hardware problems, and medical complications, which is also in line with previous cohorts. ${ }^{15-19}$ In our cohort, HwF was observed exclusively in the monocortical placement of lateral mass screws in the cervical segment. Patients undergoing anterior corpectomy and $360^{\circ}$ fusion showed no $\mathrm{HwF}$ at a mean follow-up of 13 months. Therefore, we recommend the use of either pedicle screws or bicortical lateral mass screws in the cervical spine.

To characterize the choice of approach and stabilization technique, this study concentrates in terms of spinal stability on bone infiltration and osteolysis according to the SINS. ${ }^{6,21}$ Cervicothoracic spinal malalignment and sagittal imbalance, like the loss of cervical lordosis, are important factors that may prompt $\mathrm{HwF}$ and need to be considered in the choice of surgical strategy. ${ }^{22-24}$

Our data show that the increased use of spinal navigation has led to an increasing use of technically challenging constructs/screws (i.e., pedicle screws in the cervical spine) while the surgical complication rate has remained constant. The use of spinal navigation seems to shift the indication toward more complex/demanding constructs, which are at the same time associated with higher mechanical load capacity. ${ }^{16,25-27}$ From an oncological point of view, the demands on surgical results have shifted from palliative pain control and the reduction or prevention of neurological deficits to durable mechanical reconstruction for cases with prolonged follow-up. This paradigm change is mostly driven by two aspects. First, the introduction of new therapies such as immunotherapeutics has increased statistical life expectancy significantly. ${ }^{28-36}$ Second, close radiological and clinical follow-up of cancer patients is leading to earlier detection of metastatic spine disease. Both of these factors are reflected by long-term survivors and the comparably low percentage of patients suffering from neurological deficits (50\%) in our cohort by the time of surgery compared to those in earlier studies..$^{14,15,19}$

\section{Conclusions}

Our data show that the management of metastatic spine disease at the CTJ is complex and that the overall complication rate is high. Posterior decompression without instrumentation should be reserved for patients with a stable SINS and advanced tumor stage because of the risk of secondary deformity. Anterior fusion seems feasible in selected cases. Posterior decompression and fusion with or without $360^{\circ}$ reconstruction are the most frequently used techniques. Care should be taken to choose instrumentation techniques that offer the highest possible biomechanical load-bearing capacity to avoid HwF. The increasing use of spinal navigation is shifting operative strategies toward more demanding constructs, while surgical complication rates remain stable. Considering the high overall complication rate, the prevention of in-hospital complications seems crucial to reduce in-hospital mortality.

\section{References}

1. Barzilai O, Fisher CG, Bilsky MH. State of the art treatment of spinal metastatic disease. Neurosurgery. 2018;82(6): 757-769.
2. Laufer I, Rubin DG, Lis E, et al. The NOMS framework: approach to the treatment of spinal metastatic tumors. Oncologist. 2013;18(6):744-751.

3. Fisher CG, DiPaola CP, Ryken TC, et al. A novel classification system for spinal instability in neoplastic disease: an evidence-based approach and expert consensus from the Spine Oncology Study Group. Spine (Phila Pa 1976). 2010; 35(22):E1221-E1229.

4. Ivanishvili Z, Fourney DR. Incorporating the Spine Instability Neoplastic Score into a treatment strategy for spinal metastasis: LMNOP. Global Spine J. 2014;4(2):129-136.

5. Campos M, Urrutia J, Zamora T, et al. The Spine Instability Neoplastic Score: an independent reliability and reproducibility analysis. Spine J. 2014;14(8):1466-1469.

6. Fisher CG, Versteeg AL, Schouten R, et al. Reliability of the spinal instability neoplastic scale among radiologists: an assessment of instability secondary to spinal metastases. AJR Am J Roentgenol. 2014;203(4):869-874.

7. Fehlings MG, David KS, Vialle L, et al. Decision making in the surgical treatment of cervical spine metastases. Spine (Phila Pa 1976). 2009;34(22)(suppl):S108-S117.

8. Ahmed AK, Goodwin CR, Heravi A, et al. Predicting survival for metastatic spine disease: a comparison of nine scoring systems. Spine J. 2018;18(10):1804-1814.

9. Luksanapruksa P, Buchowski JM, Hotchkiss W, et al. Prognostic factors in patients with spinal metastasis: a systematic review and meta-analysis. Spine J. 2017;17(5):689-708.

10. Choi D, Fox Z, Albert T, et al. Prediction of quality of life and survival after surgery for symptomatic spinal metastases: a multicenter cohort study to determine suitability for surgical treatment. Neurosurgery. 2015;77(5):698-708.

11. Polly DW Jr, Chou D, Sembrano JN, et al. An analysis of decision making and treatment in thoracolumbar metastases. Spine (Phila Pa 1976). 2009;34(22)(suppl):S118-S127.

12. Tokuhashi Y, Uei H, Oshima M, Ajiro Y. Scoring system for prediction of metastatic spine tumor prognosis. World J Orthop. 2014;5(3):262-271.

13. Tomita K, Kawahara N, Kobayashi T, et al. Surgical strategy for spinal metastases. Spine (Phila Pa 1976). 2001;26(3): 298-306.

14. An HS, Vaccaro A, Cotler JM, Lin S. Spinal disorders at the cervicothoracic junction. Spine (Phila Pa 1976). 1994;19(22): 2557-2564.

15. Le H, Balabhadra R, Park J, Kim D. Surgical treatment of tumors involving the cervicothoracic junction. Neurosurg Focus. 2003;15(5):E3.

16. Theologis AA, Burch S. Safety and efficacy of reconstruction of complex cervical spine pathology using pedicle screws inserted with stealth navigation and 3D image-guided (O-arm) technology. Spine (Phila Pa 1976). 2015;40(18):1397-1406.

17. Kreshak JL, Kim DH, Lindsey DP, et al. Posterior stabilization at the cervicothoracic junction: a biomechanical study. Spine (Phila Pa 1976). 2002;27(24):2763-2770.

18. Quan GM, Vital JM, Pointillart V. Outcomes of palliative surgery in metastatic disease of the cervical and cervicothoracic spine. J Neurosurg Spine. 2011;14(5):612-618.

19. Mazel C, Hoffmann E, Antonietti P, et al. Posterior cervicothoracic instrumentation in spine tumors. Spine (Phila Pa 1976). 2004;29(11):1246-1253.

20. Bayerl SH, Pöhlmann F, Finger T, et al. Two-level cervical corpectomy-long-term follow-up reveals the high rate of material failure in patients, who received an anterior approach only. Neurosurg Rev. 2019;42(2):511-518.

21. Versteeg AL, van der Velden JM, Verkooijen HM, et al. The effect of introducing the spinal instability neoplastic score in routine clinical practice for patients with spinal metastases. Oncologist. 2016;21(1):95-101.

22. Ames CP, Smith JS, Eastlack R, et al. Reliability assessment of a novel cervical spine deformity classification system. $J$ Neurosurg Spine. 2015;23(6):673-683. 
23. Lau D, Winkler EA, Than KD, et al. Laminoplasty versus laminectomy with posterior spinal fusion for multilevel cervical spondylotic myelopathy: influence of cervical alignment on outcomes. J Neurosurg Spine. 2017;27(5):508-517.

24. Kennamer BT, Arginteanu MS, Moore FM, et al. Complications of poor cervical alignment in patients undergoing posterior cervicothoracic laminectomy and fusion. World Neurosurg. 2019;122:e408-e414.

25. Mason A, Paulsen R, Babuska JM, et al. The accuracy of pedicle screw placement using intraoperative image guidance systems. J Neurosurg Spine. 2014;20(2):196-203.

26. Fehlings MG, Ahuja CS, Mroz T, et al. Future advances in spine surgery: the AOSpine North America perspective. Neurosurgery. 2017;80(3S):S1-S8.

27. Kothe R, Rüther W, Schneider E, Linke B. Biomechanical analysis of transpedicular screw fixation in the subaxial cervical spine. Spine (Phila Pa 1976). 2004;29(17):1869-1875.

28. Luke JJ, Flaherty KT, Ribas A, Long GV. Targeted agents and immunotherapies: optimizing outcomes in melanoma. Nat Rev Clin Oncol. 2017;14(8):463-482.

29. Rosenblatt J, Avigan D. Cellular immunotherapy for multiple myeloma. Cancer J. 2019;25(1):38-44.

30. Castella M, Fernández de Larrea C, Martín-Antonio B. Immunotherapy: a novel era of promising treatments for multiple myeloma. Int J Mol Sci. 2018;19(11):E3613.

31. Xia L, Liu Y, Wang Y. PD-1/PD-L1 blockade therapy in advanced non-small-cell lung cancer: current status and future directions. Oncologist. 2019;24(suppl 1):S31-S41.

32. Yoneda K, Imanishi N, Ichiki Y, Tanaka F. Immune checkpoint inhibitors (ICIs) in non-small cell lung cancer (NSCLC). J UOEH. 2018;40(2):173-189.

33. Esteva FJ, Hubbard-Lucey VM, Tang J, Pusztai L. Immunotherapy and targeted therapy combinations in metastatic breast cancer. Lancet Oncol. 2019;20(3):e175-e186.

34. Mansour M, Teo ZL, Luen SJ, Loi S. Advancing immunotherapy in metastatic breast cancer. Curr Treat Options Oncol. 2017;18(6):35.

35. Sosman JA, Kim KB, Schuchter L, et al. Survival in BRAF V600-mutant advanced melanoma treated with vemurafenib. N Engl J Med. 2012;366(8):707-714.

36. Long GV, Hauschild A, Santinami M, et al. Adjuvant dabrafenib plus trametinib in stage III BRAF-mutated melanoma. N Engl J Med. 2017;377(19):1813-1823.

\section{Disclosures}

Dr. Eicker receives honoraria from Stryker and Spineart. Dr. Thomé is a consultant for and receives honoraria from Brainlab, DePuy Synthes, Intrinsic Therapeutics, and Signus Medical; receives honoraria from Icotec, Medtronic, NuVasive, Siemens, and Zeiss; and receives non-study-related support from Brainlab, DePuy Synthes, Icotec, Intrinsic Therapeutics, Pfizer, Signus Medical, and TETEC. Dr. Meyer is a consultant for Icotec, Medtronic, DePuy, Ulrich, Spineart, Medacta, and Brainlab.

\section{Author Contributions}

Conception and design: Onken, Hubertus, Vajkoczy, Meyer. Acquisition of data: all authors. Analysis and interpretation of data: Onken, Hubertus, Mariño, Sommer, Eicker, Stangenberg, Janssen, Wipplinger, Wagner, Lange, Jörger, Vajkoczy, Meyer. Drafting the article: Onken, Hubertus, Mariño, Vajkoczy, Meyer. Critically revising the article: Onken, Gempt, Sommer, Eicker, Stangenberg, Dreimann, Janssen, Wipplinger, Wagner, Lange, Jörger, Czabanka, Rohde, Schaller, Thomé, Vajkoczy, Meyer. Reviewed submitted version of manuscript: Onken. Approved the final version of the manuscript on behalf of all authors: Onken. Statistical analysis: Onken, Hubertus. Administrative/technical/ material support: Onken, Vajkoczy, Meyer. Study supervision: Onken, Vajkoczy, Meyer.

\section{Supplemental Information}

\section{Previous Presentations}

This work was presented as an oral presentation at the 71st Annual Meeting of the German Society of Neurosurgery (DGNC) held on June 21-24, 2020, in Lübeck, Germany, at the Annual Meeting of the Department of Neurooncology of the German Society of Neurosurgery (DGNC, STNO) held on October 18, 2020, in Berlin, Germany, and at the 15th Annual Meeting of the German Spine Society (DWG) held on December 10-12, 2020, in Berlin, Germany.

\section{Correspondence}

Julia S. Onken: Charité-Universitätsmedizin Berlin, Germany. julia.onken@charite.de. 